




\section{Sumário}

Dossiê Temático: Direito Internacional Econômico.........................................................1

EDITORIAL:

Resultados da III Conferência Bienal da Red DEI ................................................. 3

Michelle Ratton Sanchez-Badin (em nome da Diretoria da Red DEI), Fabio Costa Morosini e Lucas da Silva Tasquetto (em nome dos organizadores da III Conferência da Red DEI)

Direito Internacional Econômico no Brasil: QUem Somos E o QUE faZemos? EvidênCIas EMPÍRICAS DE 1994 A 2014

Michelle Ratton Sanchez Badin, Fabio Costa Morosini e Inaê Siqueira de Oliveira

UM ESPAÇO PARA PENSAR EM ALTERNATIVAS? A ACADEMIA LATINO-AMERICANA DE DIREITO INTERNACIONAL ECONÔMICO FRENTE À ORDEM ECONÔMICA GLOBAL

Nicolás Marcelo Perrone

Grupo de alto Nível Brasil - Urugua (GAN): um Novo paradigma para a INTEgraÇão PRODUTIVA NO MERCOSUL

Alebe Linhares Mesquita e Vivian Daniele Rocha Gabriel

O COMÉRCIO de SERVIÇOS ENTRE Brasil E URUGUAI: LiberalizaÇÃo, DESAFIOS E PERSPECTIVAS DO SETOR DE TECNOLOGIA DA INFORMAÇÃO E COMUNICAÇÃO (TIC) E SOFTWARES

Vivian Daniele Rocha Gabriel e Alebe Linhares Mesquita

Core Labor Standards No Regime de PREFERÊNCIAS TARIFÁrias No MERCOSUL: A NeCESSIDADE DE HUMANIZAÇÃO DO COMÉRCIO INTERNACIONAL

Martinho Martins Botelho e Marco Antônio César Villatore

ACordo TRIMS: FleXibilizaÇão ou Não? Política de CONTEÚdo loCal, PROCESSO PROdUTIVO BÁSICO (PPB) E OS DESAFIOS PARA A INDÚSTRIA BRASILEIRA E A INTEGRAÇÃo LATINO-AMERICANA 100

Natália Figueiredo 
ESTUDIOS DE CASO DE RECHAZOS EN FRONTERA DE EXPORTACIONES ALIMENTARIAS LATINOAMERICANAS POR MOTIVOS RELACIONADOS CON MEDIDAS TÉCNICAS NO ARANCELARIAS.

Sofía Boza, Juan Rozas e Rodolfo Rivers

América do Sul em face dos tratados bilaterais de inVESTimento: RUMO aO RETORNo do EsTADO NA SOLUÇão DE CONTROvÉRSIAS? 133 Magdalena Bas

FutURo DE LOS SISTEMAS NACIONALES DE CIENCIA TECNOLOGÍA E INNOVACIÓN EN LA AGENDA ECONÓMICA DE AMÉRICA LATINA: DEFINIENDO CAMBIOS REGULATORIOS O PROTEGIENDO INVERSIONES 146

Rodrigo Corredor

EL PAPEL DE LAS INSTITUCIONES DE CONTROL FINANCIERO SOBRE LOS DERECHOS HUMANOS EN EL CONTEXTO LATINOAMERICANO 157 Jose Miguel Camacho Castro

CONVERGENCIA REgUlATORIA EN LA ALIANZA DEL PACÍFICO: UN CAPÍTULO INCONCLUSO 170 Rodrigo Polanco Lazo

O CONSTITUCIONALISMO E A COMUNITARIZAÇÃO NO DIREITO INTERNACIONAL: POSSIBILIDADES PARA O COMÉRCIO INTERNACIONAL?

Camilla Capucio

ESCASSEZ HÍDRICA E DIREITO INTERNACIONAL ECONÔMICO: O BRASIL COMO PROTAGONISTA NA TRANSFERÊNCIA DE ÁGUA PARA REGIÕES ÁRIDAS

Douglas de Castro

A Segurança energética como base para maior integração na América Do Sul: à espera DE UM TRATADO MULTILATERAL

Matheus Bassani

Outros Artigos. 246

As PRÁtICAS RESTRITIVAS DA CONCORRÊNCIA NO MERCADO DE CONTRATAÇÃo PÚBLICA EUROPEU .. 248 Alice Rocha da Silva e Ruth M. P. Santos 
Do TRANSNACIONAL PARA O NACIONAL: IOSCO, O MERCADO DE VALORES MOBILIÁRIOS BRASILEIRO E ACCOUNTABILITY

Salem Nasser, Nora Rachman e Viviane Muller Prado

MigRaÇÃo de TRABALHADORES INTELECTUAIS BRASILEIROS PARA O MERCADO INTERNACIONAL: IDENTIFICAÇÃO DE ATOS DE ALICIAMENTO DE EMPREGADOS E MECANISMOS LEGAIS PARA IMPEDIR A APROPRIAÇÃo TECNOLÓGICA E CONCORRÊNCIA DESLEAL ..........................................285 José Carlos Vaz e Dias e João Marcelo Sant'Anna da Costa

THE EASIER WAY TO HAVE "BETTER LAW"? THE MOST-SIGNIFICANT-RELATIONSHIP DOCTRINE AS THE FALLBACK CONFLICT-OF-LAW RULE IN THE PEOPLE'S REPUBLIC OF CHINA

Chi Chung

REFLEXOS JURÍDiCOS DA GOVERNANÇA GLOBAL SUBNACIONAL: A PARADIPLOMACIA E O DIREITO INTERNACIONAL: DESAFIO OU ACOMODAÇÃO 320

Valéria Cristina Farias e Fernando Rei

Matrizes Políticas da JUSTiÇA PENAL INTERNACIONAL. 341 Francisco Rezek

RESPONSABILIDAD INTERNACIONAL DEL ESTADO FRENTE A LUCHA CONTRA LA DISCRIMINACIÓN RACIAL Y ÉTNICA EN ESPAÑA. 348

Edilney Tomé da Mata e Eduardo Biacchi Gomes Correio

The Peace Process in Sierra Leone: an analysis on marriages between culture and CRIME

Gustavo Bussmann Ferreira

Funcionalização e expansão do Direito Penal: o Direito Penal negocial ...............376 Antonio Henrique Graciano Suxberger e Dermeval Farias Gomes Filho

Proteção Internacional do Consumidor e Cooperação Interjurisdicional .396 Héctor Valverde Santana e Sophia Martini Vial

The land rights of indigenous and traditional peoples in Brazil and Australia.... 418 Márcia Dieguez Leuzinger e Kylie Lyngard 
The reception of European ideas in Latin America: the issue of the German sources in Tobias Barreto, A Prominent nineteenth CENTURy BraZilian legal scholar........439 Arnaldo Sampaio de Moraes Godoy

Normas Editoriais ..................................................................................... 461 


\title{
Escassez hídrica e direito internacional econômico: o Brasil como protagonista na transferência de água para regiões áridas*
}

\author{
Water scarcity and international economic \\ law: Brazil as a key-actor in transfering water to \\ arid regions
}

Douglas de Castro**

\section{Resumo}

O artigo tem por objetivo geral operacionalizar o conceito de água virtual com base na revisão de literatura especializada para, em seguida, aproximá-lo do direito internacional econômico com o objetivo específico de apresentar as implicações de natureza econômica e ambiental no comércio internacional de produtos agrícolas e a sua contribuição para diminuir a escassez hídrica em regiões áridas. Para tanto, apresentaremos o conceito de água virtual como originalmente formulado por Anthony Allan e a posterior incorporação no conceito dos indicadores de water footprint e rotulagem, que juntos apresentam um modelo com grande potencial analítico. Em seguida, abordaremos os temas associados ao comércio internacional que sofrem a influência do conceito da água virtual, enfrentando, inclusive, alegações de práticas discriminatórias no âmbito do GATT. A análise contará na sua dimensão empírica com o método qualitativo de estudo do caso brasileiro e utilização de técnica de process tracing, sendo justificada a escolha do caso como um typical case pelo protagonismo internacional do Brasil na exportação de bens agrícolas vis-à-vis as responsabilidades assumidas em temas ambientais. Conclui-se da análise do caso que o Brasil reúne as condições internas e externas para a utilização do conceito de água virtual como vantagem comparativa em foros multilaterais para ampliar o fluxo de água que "escorre" no comércio internacional e contribuir para a redução dos efeitos da escassez hídrica em países áridos. Com este estudo espera-se contribuir para o diálogo entre os dois temas que têm recebido pouca atenção no Brasil.

Palavras-chave: Água virtual. Comércio internacional. Escassez hídrica.

* Recebido em 23/03/2016

Aprovado em 08/05/2016

** Douglas de Castro, LL.M. pela Brigham Young University, Mestre em Direito Internacional pela Faculdade de Direito da Universidade de São Paulo, Doutor em Ciência Política pela Faculdade de Filosofia, Letras e Ciências Humanas da Universidade de São Paulo, Pós-Doutorando em Direito Internacional Econômico pela Escola de Direito de São Paulo da Fundação Getúlio Vargas. E-mail: douggcastro@gmail.com.

\section{Abstract}

This article has the objective to operationalize the concept of virtual water drawing from the literature review and then move it close to the international economic law with the specific objective of presenting the economic and environmental implications in international trade of agricultural products and its contribution to reduce water scarcity in arid regions. The- 
refore, we will introduce the concept of virtual water as originally formulated by Anthony Allan and the incorporation into the concept of the water footprint and labeling indicators, which together present a model with great analytical potential. Then we discuss the issues related to international trade that are influenced by the concept of virtual water, including allegations of discriminatory practices under the GATT. The analysis in its empirical dimension will use the qualitative method of case study of the Brazilian case and will use the process tracing technique, justifying the case selection as a typical one in regards to the international role of Brazil in exporting agricultural goods vis-à-vis the responsibilities assumed on environmental issues. It is clear from the analysis that Brazil joins internal and external conditions for the application of the concept of virtual water as comparative advantage in multilateral forums to increase the flow of water in international trade and contribute to reducing the effects water scarcity in arid countries. This study is expected to contribute to the dialogue between these two areas that have received little attention in Brazil.

Keywords: Virtual water. International trade. Water scarcity.

\section{INTRODUÇão}

A água é um dos elementos mais importantes para manutenção da vida no planeta. No entanto, a sua distribuição desigual coloca algumas regiões em situação de grave stress hídrico, portanto, tornando-se uma questão de política pública a necessidade de se fixar prioridades para que o uso da água seja sustentável, viabilizando, assim, a sobrevivência dos seres humanos e continuidade de atividades a ela associadas.

Dentre os usos que são feitos da água, a utilização na agricultura e pecuária geram implicações importantes ante a crescente demanda por alimentos em quantidade e qualidade suficientes para satisfazer as necessidades básicas. Ocorre que não obstante sua importância, a agricultura e pecuária são atividades antrópicas que mais consomem e poluem as fontes de água no planeta, especialmente nos processos altamente dependentes de irrigação.

Desse modo, em razão destes cenários contraditórios questiona-se: como conciliar em países onde há a presença de escassez hídrica a necessidade de priorizar os usos da água com a produção de alimentos, ambos elementos essenciais para a realização dos direitos humanos?

A resposta para esse questionamento passa primeiramente pelo reconhecimento da premissa básica que em uma situação de stress hídrico a prioridade recairá sobre o uso que garanta a sobrevivência humana. Por outro lado, a sobrevivência humana também demanda o acesso a alimentos em qualidade e quantidade suficientes para se atingir a adequada segurança alimentar.

O enfrentamento dessa problemática passa pela adoção de uma abordagem que contemple duas atividades próprias do ser humano: o exercício da criatividade para resolução de problemas e a produção do direito.

Sob o ponto de vista de uma abordagem criativa, a utilização do conceito da água virtual tornaria possível equacionar os usos da água que concorrem com a agricultura em regiões áridas.

O conceito operacional de água virtual está ligado à transferência da água utilizada na produção de alimentos de uma região para outra pela via do comércio internacional. Neste processo há uma compensação de disponibilidade de água entre as regiões, ou seja, os produtos com alta demanda de uso de água na sua produção seriam importados, fazendo, assim, com que a prioridade de utilização da água recaia sobre àqueles ligados diretamente a sobrevivência e, portanto, mitigando os efeitos da escassez hídrica. Na outra ponta, os países com água em abundância passariam a transferir a água consumida na produção de alimentos pela sua exportação para países áridos, atribuindo, assim, valor econômico para a água.

Estima-se, conforme se comprovará mais adiante, que a água virtual que "escorre" pela via do comércio internacional alcança o volume anual de 2 bilhões de metros cúbicos, ou seja, o volume anual de grandes rios internacionais como o Congo e Ganges. Nesse ponto, a dimensão do direito internacional econômico torna-se indispensável como regulador de fluxos de produtos altamente dependentes de água para regiões do globo que não podem produzi-los em detrimento de usos essenciais para a própria sobrevivência do homem e animais, trazendo, assim, implicações importantes para enfrentar os efeitos negativos da escassez hídrica e, por consequência, incrementar a segurança hídrica e alimentar nestes países. 
A condução da análise proposta neste trabalho será feita com base no modelo criatividade-direito internacional econômico conforme exposto acima a partir de uma metodologia qualitativa de pesquisa que buscará encontrar as causas determinantes que sustentam o modelo. Utilizaremos o método de abordagem hipotético-dedutivo que será norteado pela seguinte hipótese de pesquisa: a inserção do conceito de água virtual no contexto e prática do comércio internacional possui implicações importantes para a redução dos efeitos negativos da escassez hídrica em regiões áridas ao mesmo tempo que enfrenta o problema da tragédia dos comuns ao atribuir uma dimensão econômica à água.

Como método de procedimento de análise utilizaremos o estudo de caso e como técnica de pesquisa o process tracing. Justifica-se a sua adoção pelo objetivo de identificar na análise do caso os elementos e causas que o modelo criatividade-direito internacional econômico apresenta a partir da hipótese formulada, que por sua vez somente podem ser captadas pela micro-observação utilizando a técnica de process tracing.

O caso que se adota para estudo é o do Brasil por razões teóricas e práticas. $\mathrm{Na}$ dimensão teórica, sua escolha segue o critério de typical case para a teoria, ou seja, o caso brasileiro apresenta a abundância em água e na produção de commodities com alta demanda de utilização de água. Na dimensão prática, há grande disponibilidade de fontes primárias de pesquisa que sugerem a participação do Brasil na transferência de água para regiões áridas pelo comércio internacional.

A estrutura do trabalho contará com o debate sobre os pressupostos teóricos do conceito de água virtual e sua relação com o comércio internacional de produtos agrícolas, apresentando uma revisão de literatura que tem por finalidade operacionalizar os conceitos e apontar seus pontos de intersecção para a formação da nova teoria contida na hipótese, para que então possa ser testada empiricamente no caso brasileiro.

O objetivo geral do trabalho a partir das considerações feitas é apontar as implicações e pressupostos da aplicação do conceito de água virtual no direito internacional econômico e a sua potencial contribuição para redução dos efeitos negativos da escassez hídrica.

Como objetivos específicos se buscará apresentar evidências minimamente suficientes que possam confirmar a hipótese, apresentar as limitações da pesquisa e indicar condições para a formação de uma agenda de pesquisa que possa avançar na temática.

Ademais, não se pretende esgotar neste trabalho as dimensões teórica e empírica sobre a aplicação da água virtual no comércio internacional, mas, outrossim, apresentar uma contribuição inovadora e significativa para o acúmulo de conhecimento sobre a temática, cujo modelo teórico aqui proposto é pouco explorado na área do direito internacional econômico e ambiental.

\section{Desenvolvimento}

\subsection{Produção agrícola e o conceito de água virtual}

Não se pode falar em água sem relacioná-la à própria história da humanidade e nos aspectos culturais, políticos, sociais e econômicos do seu desenvolvimento. A necessidade de ordenar o uso da água para a agricultura é, sem dúvida, a mais antiga forma de racionalizar a sua utilização. ${ }^{1}$

O desenvolvimento de técnicas de cultivo e irrigação de culturas foi o grande responsável pelo desenvolvimento das civilizações ribeirinhas, especialmente nos rios Nilo, Tigre-Eufrates, Indo, Amarelo e Yangtzé, sem contar as antigas civilizações da América Central e do Sul. ${ }^{2}$

$\mathrm{Na}$ esteira do desenvolvimento da humanidade e em relação à crescente necessidade de bens e serviços essenciais para a manutenção da vida humana, como especial menção à necessidade de alimentação, os processos de irrigação respondem, mundialmente, por pelo menos $70 \%$ do consumo total de água em atividades antrópicas. ${ }^{3}$ Adicione-se ao necessário uso da água nessas atividades as fontes de poluição que passam a preocupar cada vez mais, especialmente no tocante à contaminação das águas subterrâneas. ${ }^{4}$

1 REBOUÇAS, Aldo da Cunha; Tundisi, José Galizia (Coord.). Águas doces no Brasil. São Paulo: Escrituras, 1999. p. 10.

2 TECLAFF, Ludwik A. The river basin in history and law. Haia: Martinus Nijhoff, 1967. p.22.

3 CLARKE, Tony; MAUDE, Barlow. Ouro azul: como as grandes corporações estão se apoderando. São Paulo: M. Books, 2003. p. 5; REBOUÇAS, Aldo da Cunha; Tundisi, José Galizia (Coord.). Águas doces no Brasil. São Paulo: Escrituras, 1999. p. 121; UNITED NATIONS WORLD WATER ASSESSMENT PROGRAMME. Water for people, water for life. New York: Berghahn Books, 2003. p. 17. 4 HOMER-DIXON, Thomas. The ingenuity gap: facing the economic, environmental, and other challenges of an increasingly com- 
Nesse contexto de alto crescimento populacional, aumento da demanda pela produção de alimentos e o surgimento de novas fontes de poluição versus a limitação física do estoque de água disponível no planeta, surge o conceito de água virtual. ${ }^{5}$

O conceito de água virtual é atribuído a Anthony Allan, que sistematiza os principais elementos do conceito e destaca a crescente necessidade de água decorrente do crescimento populacional e do padrão de consumo de alimentos. Segundo o autor, a água virtual é "the water required to produce the food, goods and services that we consume daily". ${ }^{6}$ Ainda segundo o autor, à água é atribuído o adjetivo virtual porque:

$[\ldots]$ once the wheat is grown, the real water used to grow it is no longer actually contained in the wheat. The concept of virtual water helps us realize how much water is needed to produce different goods and services. In semi-arid and arid areas, knowing the virtual water value of a good or service can be useful towards determining how best to use the scarce water available.

A produção de bens e serviços dependem do uso da água em maior ou menor escala. A água utilizada na produção agrícola chama-se virtual, pois, em geral, para a produção de 1 quilo de grãos, é necessário o uso do equivalente a 1-2 $\mathrm{m}^{3}$ (1.000-2.000 litros) de água, com as variações atribuídas ao produto segundo a época do plantio, se foi cultivado em país árido ou em país com alta umidade, eficiência do uso da água em processo de irrigação, dentre outros. ${ }^{8}$

Assim, as transferências de bens e serviços entre os países formam " $[\ldots]$ communities and nations that live in river basins (watersheds) operate in "open" economic systems (problem sheds) where resource shortages

plex and unpredictable future. New York: Vintage, 2002. p. 347-348. 5 Digno de nota é o nexo entre a produção de alimentos e o consumo de petróleo, bem como a produção agrícola de cana de açúcar para produção de etanol como uma fonte alternativa de energia. Nesta última dimensão o conceito de água virtual pode ser aplicado com grande vantagem. Lester Brown aponta que "Producing ethanol at 60c per gallon, Brazil is in a strong competitive position in a world with \$60-a-barrel oil”". BROWN, Lester R. Plan B 2.0: rescuing a planet under stress and a civilization in trouble. London: Exp Upd Edition, 2006. p.32.

6 ALLAN, Tony. Fortunately there are substitutes for water otherwise our hydro-political futures would be impossible. Priorities for water resources allocation and management, London, p. 13-26, 1993. p. 14. 7 ALLAN, Tony. Virtual water: tackling the threat to our planet's most precious resource. London; New York: I. B. Tauris, 2011. p. 15. 8 HOEKSTRA, Arjen Y.; CHAPAGAIN, Ashok K. Globalization of water: sharing the planet's freshwater resources. Malden: WileyBlackwell, 2008. p. 26. can be compensated".

No entanto, o conceito passou a ter uma maior utilização e ganhou prestígio perante a comunidade científica com a agenda de pesquisa conduzida por A. Y. Hoekstra da Universidade de Twente, Holanda e pela UNESCO-IHE Institute for Water Education, que realizaram investigação e quantificação dos fluxos de comércio da água virtual entre os países, tornando o conceito operacional. ${ }^{10}$

Embora o conceito seja relativamente simples, ele possui importantes implicações para as dimensões do meio ambiente, política, economia e social de um país, região e globo como um todo. ${ }^{11}$ Segundo Marta Antonelli e Francesca Greco:

$[\ldots]$ most of the water we use is the water we "eat", that is the water contained (even if we cannot see it) in the food that arrives on our table after having gone through the various phases of production, transformation and distribution. ${ }^{12}$

A água virtual é a soma dos conceitos de green water e blue water desenvolvido por Malin Falkenmark ${ }^{13}$, que representa, respectivamente, o volume da água que evapora durante a fase de cultivo e o volume bombeado e usado na fase de cultivo, que deve, ainda, ser somada à água necessária para a dispersão de poluentes utilizados no processo. ${ }^{14}$

O anexo I mostra a racionalidade da utilização do conceito de água virtual: os países que integram uma bacia hidrográfica internacional localizada em uma região árida devem priorizar o uso da água na produção de bens e serviços que não demandem grandes quantidades de água. Como se pode verificar no anexo, um ham-

9 ALLAN, Tony. Virtual water: tackling the threat to our planet's most precious resource. London; New York: I. B. Tauris, 2011. p. 16. 10 HOEKSTRA, Arjen Y.; HUNG, P. Q. Virtual water trade: a quantification of virtual water flows between nations in relation to international crop trade. Value of Water Research Report Series, n. 11, p. 25-47, Sept. 2002. p. 37.

11 ALLAN, Tony. Virtual water: tackling the threat to our planet's most precious resource. London; New York: I. B. Tauris, 2011. p. 1. 12 ANTONELLI, Marta; GRECO, Francesca. The water we eat: combining virtual water and water footprints. New York: Springer, 2015. p. 156.

13 FALKENMARK, Malin. The massive water scarcity now threatening Africa: why isn't it being addressed? Ambio, v. 18, n. 2, p. 112-118, 1989. p.116.

14 HOEKSTRA, Arjen Y.; CHAPAGAIN, Ashok K.; ALDAYA, M.M.; MEKONNEN, M. M. Water footprint assessment manual: the global standard. Avaiable at: </en/resources/publications/waterfootprint-assessment-manual-global-standard/>. Accessed: 6 May 2016. 
búrguer contém 2.400 litros de água virtual, pois leva-se em consideração os processos complexos de produção e distribuição de um simples sanduíche..$^{15} \mathrm{~A}$ revista The Economist estima que nos Estados Unidos são consumidos anualmente 40 bilhões de hambúrgueres, que multiplicados pelos 2.400 litros nos mostra um cenário com implicações importantes. ${ }^{16}$

Nesses países é fundamental como política pública priorizar os usos que atendam às necessidades fundamentais do ser humano e a manutenção do ecossistema em que ele está inserido para atender requisitos de qualidade de vida ${ }^{17}$. Por outro lado, na dimensão da segurança alimentar, o reconhecimento dessas prioridades implica negociar importações de bens e serviços com os países que tenham recursos hídricos em abundância. Um relevante estudo de caso aponta para a confirmação dessa proposição:

\footnotetext{
In France, for example, 530 litres of water are needed to produce one kilogram of maize in irrigated farming, as against 1,100 litres in Egypt. These differences occur basically because of the higher evapotranspiration rates in hot, arid and semi-arid regions compared to cooler regions with. ${ }^{18}$
}

Assim, o conceito de água virtual promove uma conexão entre a água, alimentos e comércio que segundo Hoekstra "can be an instrument to increase global water use efficiency" em termos de distribuição da água

15 Para efeitos do presente trabalho e dada a complexidade do tema deixaremos de analisar a participação das empresas transnacionais de alimentos e grandes redes de supermercados na transferência da água virtual, reservando a análise à esfera pública de regulação do comércio internacional que é anterior às transações privadas. Com isso não tiramos a importância da participação de grandes empresas no processo que certamente devem fazer parte da agenda de pesquisa sobre água virtual. Ver ANTONELLI, Marta; GRECO, Francesca. The water we eat: combining virtual water and water footprints. New York: Springer, 2015. p. 49-50.

16 AS HAMBURGUERS go, so goes America? The Economist, 21 Aug. 1997. Available at: <http://www.economist.com/ node/154515>. Accessed: 17 Apr. 2016.

17 A escassez de água em alguns países, de fato, pode levar a escolhas políticas para restringir a exportação de alimentos. O governo de Israel, por exemplo, desencoraja a exportação de laranjas — tradicionalmente cultivadas com um sistema de irrigação pesado —, para evitar que grandes quantidades de água virtual sejam exportadas para diferentes partes do mundo. LOBO, Thaís. Brasil exporta cerca de 112 trilhões de litros de água doce por ano. O Globo, 11 set. 2012. Ciência. Disponível em: <http://oglobo.globo.com/sociedade/ciencia/brasil-exporta-cerca-de-112-trilhoes-de-litros-deagua-doce-por-ano-6045674>. Acesso em: 17 abr. 2016.

18 HORLEMANN, Lena; NEUBERT, Susanne. Virtual water trade: a realistic concept for resolving the water crisis? Bonn: Deutsches Institut für Entwicklungspolitik, 2007.
.${ }^{19}$ Nesse mesmo sentido, Lena Horlemann e Susanne Neubert do German Development Institute apresentam implicações que não podem ser desprezadas por acadêmicos e policy-makers:

Globally, agricultural trade is already resulting in the "saving" of some $455 \mathrm{~km}^{3}$ of water each year, which is equivalent to about $8 \%$ of water resources compared to local consumption. It thus appears that the potential savings if strategic Virtual Water Trade is introduced consistently are enormous, since the more products produced in use-efficient countries, the more water "saved" globally. ${ }^{20}$

Como se percebe, a estratégia de utilização da água virtual em produtos agrícolas possui um importante potencial aumentar a sustentabilidade dos usos da água. ${ }^{21}$ No entanto, ainda que sob o ponto de vista lógico, a utilização da água virtual seja um dos meios de solução da má distribuição da água no planeta, ela esbarra em considerações de natureza política e econômicas pautadas pelos interesses nacionais que impõem custos transacionais para os países como se observa nas negociações travadas na Rodada de Doha ${ }^{22}$, o que vale dizer que a utilização do conceito de água virtual no comércio internacional assume, também, uma dimensão política, pois:

Political decision-makers actively pressing for the increased import of agricultural products in order to conserve water resources at national level. According to Allan, the concept is suitable as the basis of a political strategy, since "serious local water shortages can be very effectively ameliorated by global economic processes. ${ }^{23}$

\section{2. Água virtual e direito internacional econômico}

Tradicionalmente, o debate sobre a liberalização do comércio de produtos agrícolas está associado a duas dimensões: a importância da agricultura nos países em

19 HOEKSTRA, Arjen Y.; CHAPAGAIN, Ashok K. Globalization of water: sharing the planet's freshwater resources. Malden: WileyBlackwell, 2008. p. 78.

20 HORLEMANN, Lena; NEUBERT, Susanne. Virtual water trade: a realistic concept for resolving the water crisis? Bonn: Deutsches Institut für Entwicklungspolitik, 2007.

21 GULBEKIAN, Think Thank. Water and the future of bumanity: revisiting water security. New York: Springer, 2013. p. 25.

22 NASH, John; MCCALLA, Alex. Reforming agricultural trade for developing countries: key issues for a pro-development outcome of the Doha round. Washington: World Bank Publications, 2006. p. 23.

23 ANTONELLI, Marta; GRECO, Francesca. The water we eat: combining virtual water and water footprints. New York: Springer, 2015. p. 56. 
desenvolvimento e o lento crescimento do comércio de produtos agrícolas dos países em desenvolvimento para os países desenvolvidos. ${ }^{24} \mathrm{O}$ debate incorpora no aspecto econômico a tensão entre os países do Norte desenvolvido e os países do Sul em desenvolvimento e no aspecto ambiental a tensão entre o desenvolvimento e proteção do meio ambiente. ${ }^{25}$ Nesse sentido Hoekstra assevera:

The WTO rules apply to most products but still exclude or include to a limited extent services and agricultural products. Because $85 \%$ of the water consumption in the world occurs in agriculture, concerns with respect to sustainable freshwater use can still be taken into account in the negotiations in the Doha Development Round, the current tradenegotiation round of the World Trade Organization which started in 2001. Trade in agricultural products is one of the key focus areas of the current negotiations. As follows from above, from a sustainable water-use perspective it is key that any new rules on international trade in agricultural products include provisions that ensure that efforts to contribute to more sustainable water use behind the products traded are promoted and rewarded. ${ }^{26}$

Ainda que se possa superar o debate que essas duas dimensões apresentam com base na premissa de que a água virtual transferida via produtos agrícolas possui grande potencial de reduzir os efeitos da escassez hídrica e aumentar a segurança hídrica e alimentar, a mera liberalização do comércio internacional com a redução de tarifas e a eliminação ou redução de subsídios não são suficientes per se para enfrentar apropriadamente a questão $^{27}$. A água e suas repercussões na produção e cultivo não é a principal preocupação que impulsiona as negociações comerciais, primeiro porque a ela não se atribui preço ou é irrisório e em segundo que outros fatores políticos mascaram sua importância. ${ }^{28}$

24 Para uma melhor compreensão do tema dos subsídios na agricultura indicamos: GOMES, Natália Fernanda. A regulação internacional dos subsídios agrícolas: a contemporaneidade do paradigma realista para a compreensão do sistema de comércio agrícola internacional vigente. Revista de Direito Internacional, v.10, n. 1, jun. 2013. doi:10.5102/rdi.v10i1.2067.

25 WEISS, Edith Brown; JACKSON, John H.; BERNASCONIOSTERWLADER, Nathalie. Reconciling environment and trade. Leiden: Brill, 2008. p. 773.

26 HOEKSTRA, Arjen Y. The relation between international trade and freshwater scarcity, Jan. 2010. Available at: <https://www.wto.org/ english/res_e/reser_e/ersd201005_e.pdf> Accessed: 17 Apr. 2016. 27 CLAPP, Jennifer; COHEN, Marc J. Global food crisis: governance challenges and opportunities. Waterloo: Wilfrid Laurier University Press, 2009. p. 43-44; SOARES, Guido Fernando Silva. Direito Internacional do Meio Ambiente: emergência, obrigações e responsabilidades. São Paulo: Atlas, 2003. p. 142-144.

28 WORLD TRADE ORGANIZATION. World Trade Report 2010: Trade in natural resources, 2010. Available at: <https://www.
Segundo Hoekstra, será necessário somar aos esforços de redução de tarifas e subsídios a adoção de mecanismos que pudessem assegurar a eficiência da transferência de água pelo comércio internacional na importação e exportação. Se de um lado busca-se mitigar os efeitos da escassez hídrica ao priorizar os usos da água e importar alimentos com alta demanda de água, por outro lado, é fundamental assegurar que o uso da água no cultivo dos produtos seja padronizado e sustentável nas dimensões econômica e ambiental. ${ }^{29}$

Citando o WTO 2010 World Trade Report para justificar a posição de que somente a redução tarifária não é suficiente para aliviar os efeitos da escassez na esfera nacional, Edith Weiss e Lydia Slobodian sugerem a adoção dos mecanismos de padronização quanto a eficiência do uso da água na produção agrícola: a adoção do footprint da água e a rotulagem que serviriam para informar o grau de eficiência de utilização da água. ${ }^{30}$

O footprint da água é um indicador que leva em consideração a utilização da água, sua poluição, clima e solo nas várias etapas do processo produtivo, distribuição e consumo, constituindo assim elemento essencial para a utilização da água virtual no comércio internacional. ${ }^{31}$

Hoekstra e Chapagain apontam os quatro maiores fatores que determinam o footprint da água deum país: 1) O volume de consumo baseado na renda média; 2) Os padrões de consumo (p.ex., relação alto $\mathrm{X}$ baixo consumo de carne); 3) As condições climáticas de cultivo; e 5) As práticas adotadas na agricultura. ${ }^{32}$

Conhecer o footprint da água por intermédio da sua inserção nos rótulos dos produtos tenderia a uma maior

wto.org/english/res_e/publications_e/wtr10_e.htm> Accessed: 05 May 2016.

29 HOEKSTRA, Arjen Y.; CHAPAGAIN, Ashok K. Globalization of water: sharing the planet's freshwater resources. Malden: WileyBlackwell, 2008. p. 34.

30 WEISS, Edith Brown; JACKSON, John H.; BERNASCONIOSTERWLADER, Nathalie. Reconciling environment and trade. Leiden: Brill, 2008. p. 775.

31 "The global water footprint is 7,450 billion $\mathrm{m} 3=\mathrm{yr}$, which is $1,240 \mathrm{~m} 3=y r$ per person on average. Humans' green water footprint in the world is 5,330 billion $\mathrm{m} 3=\mathrm{yr}$, while the blue water footprint amounts to 2,120 billion $\mathrm{m} 3=\mathrm{yr}$. All the green water goes into agricultural products; the blue water is used for agricultural products $(50 \%)$, industrial products (34\%), and domestic water services (16\%)". HOEKSTRA, Arjen Y.; CHAPAGAIN, Ashok K. Globalization of water: sharing the planet's freshwater resources. Malden: Wiley-Blackwell, 2008. p. 67.

32 HOEKSTRA, Arjen Y.; CHAPAGAIN, Ashok K. Globalization of water: sharing the planet's freshwater resources. Malden: WileyBlackwell, 2008. p. 98. 
eficiência no uso da água ao incentivar práticas sustentáveis. Nesse sentido para Weiss e Slobodian:

Water labeling in the context of the trade in virtual
water would potentially make water content a
factor in demand for certain products. Labels give
consumers especially valuable information when
they relate to attributes not immediately apparent
in the final product. Consumers can then draw
on environmental, social, and other values in
making purchasing decisions, and put pressure on
producers to conform to these values. ${ }^{33}$

Em termos de rotulagem, para além das negociações entre os Estados no âmbito da competência da OMC, vemos uma crescente tendência que terá impacto na água virtual e comércio internacional e que suplementa a atuação dos Estados que é certificação voluntária para cumprir com padrões ambientais e sociais em uma grande variedade de comanditeis, com a participação cada vez maior de atores não estatais como é o caso da International Social and Environmental Accreditation and Labelling Alliance (ISEAL). ${ }^{34}$

Desse modo, o potencial do conceito de água virtual no comércio internacional e seu papel na redução dos efeitos da escassez e do aproveitamento mais eficiente da água localmente é altamente promissor. A adoção do water footprint e rotulagem como mecanismos que garantam o uso sustentável da água apresenta um apelo político, social, econômico e ambiental que não podem ser desprezados. Ainda que possua força analítica e prática, a adoção dessas medidas, considerando a complexidade e interdependência associados ao tema, nos conduz à formula popperiana de resolução de problemas:

$$
\mathrm{P}_{1} \rightarrow \mathrm{TS} \rightarrow \mathrm{EE} \rightarrow \mathrm{P}_{2}
$$

A solução do problema inicial da liberalização $\left(\mathrm{P}_{1}\right)$ conduz à solução tentativa que é a proposta do mecanismo de aumento de eficiência do uso da água (TS), que por sua vez passa pelo processo de eliminação de erro aplicado à solução tentativa (EE) que culmina com o potencial surgimento de novos problemas associados à non-product-related process e production method distinction no âmbito do Acordo sobre Barreiras Técnicas ao Comércio $\left(\mathrm{P}_{2}\right){ }^{35}$

33 WEISS, Edith Brown; JACKSON, John H.; BERNASCONIOSTERWLADER, Nathalie. Reconciling environment and trade. Leiden: Brill, 2008. p. 780.

34 RAYNOLDS, Laura T.; MURRAY, Douglas; HELLER, Andrew. Regulating sustainability in the coffee sector: a comparative analysis of third-party environmental and social certification initiatives. Agriculture and human values, v. 24, n. 2, p. 147-163, Mar. 2007. doi:10.1007/s10460-006-9047-8. p. 151.

35 O'HEAR, Anthony. Karl Popper: philosophy and problems.
Por exemplo, um copo de suco de laranja que utiliza 170 litros de água (Anexo I) em um determinado país tem o mesmo gosto, cheiro e cor que um produzido em país com uma menor eficiência do uso da água que utilizará, p.ex., 250 litros, ou seja, os mesmos produtos deixam de ser "idênticos" sob o ponto de vista do mercado consumidor e, portanto, tornando a exigência de rotulagem e water footprint discriminatória. ${ }^{36}$

A solução do desafio que surge no bojo da necessidade de assegurar a eficiência do uso da água na agricultura, segundo Weiss e Slobodian se fundamenta juridicamente no artigo XX do GATT. ${ }^{37}$

Se, por um lado, o artigo 2.1 do Acordo sobre Barreiras Técnicas ao Comércio estabele que:

Members shall ensure that in respect of technical regulations, products imported from the territory of any Member shall be accorded treatment no less favourable than that accorded to like products of national origin and to like products originating in any other country.

Por outro lado, o artigo XX, letras "b" e "g" do GAT'T, apresenta exceções à medidas protecionistas de caráter discriminatórias que o conceito de water footprint e rotulagem possam apresentar. A letra "b" autoriza a adoção de medidas "necessary to protect human, animal or plant life or health" e a letra "g" medidas "relating to the conservation of exhaustible natural resources if such measures are made effective in conjunction with restrictions on domestic production or consumption".

Desse modo, os pseudos-desafios que o water foodprint e rotulagem representam para o comércio internacional e as negociações multilaterais são enfrentados satisfatoriamente, de forma a não impedir sua promoção e adoção.

Lançadas as bases teóricas que envolvem o conceito de água virtual e o comércio internacional, passaremos a análise empírica da participação brasileira nessas dimensões. No entanto, antes de fazê-la, é de grande importância notar que a pesquisa sobre o tema da água virtual, em especial sua relação com o comércio internacional, é

New York: Cambridge University Press, 1996. p. 28.

36 WORLD TRADE ORGANIZATION. United States: measures concerning the importation, marketing and sale of tuna and tuna products, 2012. Available at: <https://www.wto.org/english/ tratop_e/dispu_e/381abr_e.pdf>. Accessed: 05 May 2016.

37 WEISS, Edith Brown; JACKSON, John H.; BERNASCONIOSTERWLADER, Nathalie. Reconciling environment and trade. Leiden: Brill, 2008. p. 720. 
insipiente na academia brasileira, o que reforça um dos objetivos do presente estudo que é o de contribuir para a geração de conhecimento no tema e indicar linhas de pesquisa futuras. ${ }^{38}$

\subsection{A participação do Brasil na distribuição de água virtual}

Dos 10 principais produtos da pauta de exportação brasileira, 6 são agropecuários segundo o UN Comtrade, dentre os quais soja (7\%), açúcar $(5.3 \%)$ e carne $(2.8 \%){ }^{39}$ Assim, o país assume um grande protagonismo nos foros internacionais de negociações em comércio internacional, bem como em foros de natureza ambiental dado sua potencial contribuição para ampliar as discussões e a efetiva utilização do conceito de água virtual.

Na pauta de exportação brasileira temos a Europa como um dos maiores comparadores segundo o Ministério da Indústria e Comércio (MIDIC). Nos meses de janeiro e fevereiro de 2016, o Brasil exportou para a União Europeia um total de 164.460.496 quilos de café em grãos, que se multiplicado pela estimativa de água virtual utilizada em grãos, teríamos um total de 164.460.496.000 litros se considerarmos que para cada quilo utiliza-se 1.000 litros de água, o que em algumas regiões essa quantidade pode chegar ao dobro. ${ }^{40}$

Para a Ásia, segundo mercado que mais o Brasil exporta, tem o milho como o produto principal. O volume de exportação atingiu no mesmo período um

38 Em pesquisa realizada em periódicos nacionais da base Scielo utilizando o parâmetro "água virtual", encontramos dois artigos: CARMO, Roberto Luiz do; OJIMA, Andréa Leda Ramos de Oliveira; OJIMA, Ricardo; NASCIMENTO, Thais Tartalha do. Água virtual, escassez e gestão: o Brasil como grande "exportador" de água. Ambiente \& Sociedade, Campinas, v. 10, n. 1, p. 83-96, jan./jun. 2007; WARNER, Jeroen; SEBASTIAN, Antoinette; EMPINOTTI, Vanessa. Claiming (back) the land: the geopolitics of egyptian and south african land and water grabs. Ambiente \& Sociedade. v. 16, n. 2, p. 1-24, June 2013. doi:10.1590/S1414-753X2013000200002. Utilizando o mesmo parâmetro de pesquisa no website da Revista de Direito Internacional não pudemos encontrar nenhuma referência ao termo (ver <http://www.publicacoesacademicas.uniceub.br/index.php/rdi/search/advancedResults> Acesso em: 05 maio 2016). 39 UNITED NATIONS. DEPARTMENT OF ECONOMIC AND SOCIAL AFFAIRS. STATISTICS DIVISION. UN Comtrade Database, 2016. Available: <http://comtrade.un.org/data/> Accessed: 05 May 2016.

40 BRASIL. Ministério da Indústria, Comércio Exterior e Serviços. Balança comercial brasileira: mensal, 2016. Disponível em: < http://www.desenvolvimento.gov.br/sitio/interna/interna. php?area $=5 \&$ menu $=1161>$. Acesso em: 05 maio 2016. montante de 2.833.225.557 quilos, o que equivale a um total de água utilizada no processo produtivo de 2.833.225.557.000. ${ }^{41}$

Os países que formam a Organização do Países Produtores de Petróleo (OPEP), que estão localizados, na sua maioria, em região com grande escassez hídrica, importam do Brasil 2.387.112.897 quilos de milho, que encerram uma quantidade de 2.387.112.897.000 litros de água virtual. ${ }^{42}$

Segundo Carmo, se considerarmos somente a soja, carne a açúcar na exportação do Brasil para os países no mundo, teríamos um total de 50 bilhões de $\mathrm{M}^{3}$ de água virtual nesses produtos em 2005, cujos custos não são computados na venda dada uma precificação falha ou inexistente. ${ }^{43}$

Não obstante os números possam divergir, a grandeza que eles representam indicam a necessidade de definições em políticas públicas que possam atingir seu potencial em benefícios econômicos, sociais e ambientais. No entanto, esse grande potencial precisa ser visto com cautela. $\mathrm{O}$ aumento das exportações de produtos com alta demanda de água para países em regiões áridas pode auxiliar no combate aos efeitos da escassez hídrica, e nesse caso, tem o Brasil como um dos maiores protagonistas em termos de disponibilidade hídrica, clima favorável e áreas de cultivo. No entanto, como visto anteriormente, a agricultura é a atividade antrópica de maior consumo de água, principalmente em irrigação, o que coloca uma grande pressão no seu uso. Segundo Hoekstra em entrevista dada ao Jornal O Globo:

[...] atualmente, ninguém paga o preço total pelo consumo de água. A escassez e a poluição precisam ser incluídas no preço das commodities. Isso criaria um incentivo para consumir e poluir menos. Mas as legislações também podem ser melhoradas e em alguns produtos pode ser útil incluir o uso de água sustentável no rótulo. ${ }^{44}$

41 BRASIL. Ministério da Indústria, Comércio Exterior e Serviços. Balança comercial brasileira: mensal, 2016. Disponível em: $<$ http://www.desenvolvimento.gov.br/sitio/interna/interna. php?area $=5 \&$ menu $=1161>$. Acesso em: 05 maio 2016.

42 BRASIL. Ministério da Indústria, Comércio Exterior e Serviços. Balança comercial brasileira: mensal, 2016. Disponível em: <http://www.desenvolvimento.gov.br/sitio/interna/interna. php?area $=5 \&$ menu $=1161>$. Acesso em: 05 maio 2016.

43 CARMO, Roberto Luiz do; OJIMA, Andréa Leda Ramos de Oliveira; OJIMA, Ricardo; NASCIMENTO, Thais Tartalha do. Água virtual, escassez e gestão: o Brasil como grande "exportador" de água. Ambiente \& Sociedade, Campinas, v. 10, n. 1, p. 83-96, jan./ jun. 2007. p. 86-87.

44 LOBO, Thaís. Brasil exporta cerca de 112 trilhões de litros 
As medidas sugeridas pela autora atacam as principais causas do desperdício e uso não sustentável da água na agricultura no Brasil: sistemas de irrigação com vazamentos, o cultivo de sementes com alta demanda de água em solo e/ou clima não apropriado e a poluição da água e de suas fontes, como é o caso do Aquífero Guarani.

As pressões na utilização e poluição da água do Aquífero Guarani são intensas e colocam em perigo uma das principais e pristinas fontes de água doce no Cone Sul. Um levantamento feito pelo Serviço Geológico do Brasil aponta que há na porção brasileira do aquífero 610 poços artesianos cadastrados. Destes, 344 não atendem os requisitos mínimos estabelecidos pela lei (o que dizer dos poços clandestinos que, intuitivamente, não cumprem com os padrões mínimos de qualidade na utilização). Quanto à poluição, o uso de pesticidas e outros resíduos utilizados na lavoura colocam em perigo a qualidade da água. ${ }^{45}$

A preocupação é tão grande que o estado de São Paulo avançou na promoção de algumas iniciativas no sentido de enfrentar o problema das águas subterrâneas, incluindo o Aquífero Guarani (lei 9866/77 e 7663/91). Como parte da estratégia, o estado promove dentre outras medidas 1) Cria a gestão descentralizada que inclui a participação de ONGs e universidades com o objetivo de promover a participação da sociedade civil na discussão e implementação de projetos relacionados a água; 2) Reconhece ser a água é um bem público que possui valor econômico e que a sua utilização deve ser cobrada; e 3) Cria a Área de Proteção e Recuperação de Mananciais, cujo objetivo principal é estabelecer um controle criterioso da ocupação e uso das áreas de acesso de mananciais e manter o envolvimento ativo da sociedade civil em ações e programas integrados. ${ }^{46}$

de água doce por ano. O Globo, 11 set. 2012. Ciência. Disponível em: <http://oglobo.globo.com/sociedade/ciencia/brasil-exportacerca-de-112-trilhoes-de-litros-de-agua-doce-por-ano-6045674>. Acesso em: 17 abr. 2016.

45 SÃO PAULO. Secretaria do Meio Ambiente. Projeto ambiental estratégico aquíferos: síntese das atividades período 2007-2010. São Paulo: Instituto Geológico, 2011. (Cadernos do Projeto Ambiental Estratégico Aquíferos, n. 3) Disponível em: < http://www.igeologico.sp.gov.br/downloads/livros/Sintese $\% 20$ Aquiferos.pdf $>$ Acesso em: 05 maio 2016.

46 FADDEN, Sabrina Mac; OLIVEIRA, Enéas Xavier de. Captação de águas subterrâneas no Estado de São Paulo. In: MOSTRA ACADÊMICA DA UNIMEP, 6., 2008, Piracicaba. Anais... Piracicaba: UNIMEP, 2008. Disponível em: < http://www.unimep.br/ phpg/mostraacademica/anais/6mostra/5/371.pdf > Acesso em: 05
No tocante aos desafios associados à irrigação sustentável, o ponto central segundo Telles é determinar os perfis de irrigação nas regiões brasileiras: no Sul e Sudeste há grande utilização de água como complemento na produção, mas sem maiores cuidados dada relativa abundância, enquanto no Nordeste a irrigação é obrigatória para o cultivo e criação de animais. ${ }^{47}$

O aumento da produção agrícola demanda um aumento das áreas irrigadas. Segundo a FAO, a área irrigada no Brasil em 1960 era de 455.433 hectares, chegando em 2006 a 4.601.288 hectares, ou seja, um aumento significativo ainda que considerada elástica a dimensão temporal entre os dois índices. ${ }^{48}$

Essa tendência, por sua vez, imporá, cada vez mais, políticas públicas e mecanismos que interpretem, corretamente, essa demanda e apresentem soluções para garantir o uso racional e sustentável. Podemos ver essa preocupação no caso do Brasil com a adoção da Lei 9.433/97 (Política Nacional dos Recursos Hídricos), que, dentre outras disposições, institui mecanismos de democratização e descentralização das decisões sobre os usos da água nas bacias hidrográficas e operacionalização da gestão da água com a outorga de direito de uso e cobrança pelo uso. ${ }^{49}$

\section{Considerações finais}

Os elementos apresentados na análise de caso do Brasil apontam para uma posição favorável do Brasil nas dimensões internacional e nacional quanto ao aproveitamento do conceito de água virtual, que até o presente é subutilizado como um vetor de política externa pela diplomacia brasileira nos aspectos ambientais e econômicos. Embora o protagonismo brasileiro seja reconhecido nos foros multilaterais, a condução da política externa vis-à-vis a emergência de novos fenômenos políticos, sociais, econômicos e naturais, internos e ex-

maio 2016.

47 TELLES, D. D’A. Água na agricultura e pecuária. In: REBOUÇAS, A; Braga B; TUNDISI, J. G. (Orgs). Águas doces no Brasil: capital ecológico, uso e conservação. 2. ed. São Paulo: Escrituras, 1999. p. 703.

48 FOOD AND AGRICULTURE ORGANIZATION OF THE UNITED NATIONS. Aquastat. Available at: < http://www.fao. org/nr/water/aquastat/main/index.stm>. Accessed: 05 May 2016. 49 BRASIL. Lei n. 9433, de 8 de janeiro de 1997. Disponível em: <http://www.planalto.gov.br/ccivil_03/leis/L9433.htm>. Acesso em: 05 maio 2016. 
ternos, gerados pelo processo de globalização, encetam uma revisão quanto à forma de sua abordagem. Sem a incorporação de novas formas de encarar esses desafios nos vetores da política externa, os objetivos do Brasil nas relações internacionais sofrem defasagem.

A incorporação do conceito de água virtual nas negociações internacionais de comércio é um dos mecanismos que possui grande potencial para mantê-lo na vanguarda como conclusão do nosso trabalho.

O exame do modelo criatividade-direito internacional econômico que foi feito no estudo de caso, traçando os elementos essenciais para a sua viabilidade como teoria aponta para um ganho analítico e de conhecimento conforme proposto. O exame detalhado e operacionalização do conceito de água virtual que foram feitos, com base em uma revisão de literatura científica e a inclusão dos indicadores da rotulagem e water fooprint, o coloca um instrumento de transferência de água para regiões áridas que precisam estabelecer prioridades quanto ao uso da água e apresenta uma forma de controle quanto ao uso sustentável e racional da água nos países produtores, evitando, assim, o desperdício ou aumento de poluição de fontes sensíveis às atividades antrópicas como é o caso dos aquíferos.

No tocante ao direito internacional econômico, a análise serviu para identificar os possíveis entraves que a aplicação do conceito de água virtual teria nas negociações internacionais de comércio e apontou para as soluções que o próprio direito fórmula ao reconhecer a desigualdade no desenvolvimento entre os atores na relação Norte-Sul, bem como da necessidade de proteção e preservação do meio ambiente nesse contexto.

No nível da análise empírica, foram encontradas evidências importantes que apontam para um grande potencial de aplicação do conceito na formulação de políticas públicas nacionais no tocante ao estabelecimento da prioridade dos usos da água e controle de desperdício e poluição, que terão repercussão no cenário mundial nas negociações que garantam esses fluxos.

Ainda na dimensão empírica, os ganhos analíticos acima relatados sofrem substancial incremento quando se focou na sua aplicação ao caso brasileiro. Os dados apresentados apontam para o reconhecimento da pujança do Brasil na agricultura, com especial ênfase na produção de grãos, cujos principais fatores podemos citar a abundância de água, clima favorável a diversas culturas e grandes áreas de terras cultiváveis, que coloca o país entre os 5 principais produtores mundiais.
Essa posição não pode ser desprezada na condução da política externa e nas negociações de comércio internacional, pois, conforme amplamente explicado no desenvolvimento do trabalho, serviria como leverage para pressionar, cada vez mais, no sentido da liberalização do comércio internacional e entraves a ela associados, ao mesmo tempo que se adotam os mecanismos da rotulagem e water footprint, que, como vimos anteriormente, satisfaz os requisitos das obrigações assumidas pelo Brasil no âmbito do GATT' e da OMC.

O protagonismo do Brasil nos foros internacionais em matéria ambiental também é amplamente reconhecido. O país vem assumindo esse protagonismo, em razão de ações positivas, ao assumir, cada vez mais, obrigações internacionais em diversas temáticas ambientais ante a comunidade internacional, mas também em virtude das ações negativas que causam preocupação como o desmatamento da Amazônia e o aumento de níveis de poluição. Em relação à abundância de água e ao seu uso racional e sustentável na agricultura, o Brasil apresenta um quadro jurídico-institucional estável, que conta, inclusive, com iniciativas importantes de estados e municípios como se viu anteriormente.

Desse modo, pode-se concluir com elevado grau de confiança que o caso brasileiro apresenta os pressupostos teóricos de aplicação do conceito de água virtual no comércio internacional como teorizados, que colocam o país como um grande exportador de água do mundo e importante interlocutor do tema em foros econômicos e ambientais internacionais. A conclusão deste estudo ainda aponta, como se pode perceber da complexidade que a temática apresenta, a necessidade da formação de uma agenda de pesquisa que possa responder aos desafios teóricos e práticos que pouco são explorados na academia e prática política.

\section{REFERÊNCIAS BIBLIOGRÁfiCAS}

ALLAN, J. A.; MERRET, S.; LANT, C. Virtual water: the water, food, and trade nexus useful concept or misleading metaphor? IWR A Water International, v. 28, n. 1, Mar. 2003.

ALLAN, Tony. Fortunately there are substitutes for water otherwise our hydro-political futures would be impossible. Priorities for water resources allocation and management, London, p. 13-26, 1993. 
ALLAN, Tony. Virtual water: tackling the threat to our planet's most precious resource. London; New York: I. B. Tauris, 2011.

ANTONELLI, Marta; GRECO, Francesca. The water we eat: combining virtual water and water footprints. New York: Springer, 2015.

AS HAMBURGUERS go, so goes America? The Economist, 21 Aug. 1997. Available at: < http://www.economist.com/node/154515>. Accessed: 17 Apr. 2016.

BRASIL. Lei n. 9433, de 8 de janeiro de 1997. Disponível em: <http://www.planalto.gov.br/ccivil_03/leis/ L9433.htm>. Acesso em: 05 maio 2016.

BRASIL. Ministério da Indústria, Comércio Exterior e Serviços. Balança comercial brasileira: mensal, 2016. Disponível em: <http://www.desenvolvimento.gov.br/sitio $/$ interna $/$ interna.php?area $=5 \&$ menu $=1161>$. Acesso em: 05 maio 2016.

BROWN, Lester R. Plan B 2.0: rescuing a planet under stress and a civilization in trouble. London: Exp Upd Edition, 2006.

CARMO, Roberto Luiz do; OJIMA, Andréa Leda Ramos de Oliveira; OJIMA, Ricardo; NASCIMENTO, Thais Tartalha do. Água virtual, escassez e gestão: o Brasil como grande "exportador" de água. Ambiente \& Sociedade, Campinas, v. 10, n. 1, p. 83-96, jan./jun. 2007. CLAPP, Jennifer; COHEN, Marc J. Global food crisis: governance challenges and opportunities. Waterloo: Wilfrid Laurier University Press, 2009.

CLARKE, Tony; MAUDE, Barlow. Ouro azul: como as grandes corporações estão se apoderando. São Paulo: M. Books, 2003.

FADDEN, Sabrina Mac; OLIVEIRA, Enéas Xavier de. Captação de águas subterrâneas no Estado de São Paulo. In: MOSTRA ACADÊMICA DA UNIMEP, 6., 2008, Piracicaba. Anais... Piracicaba: UNIMEP, 2008. Disponível em: <http://www.unimep.br/phpg/mostraacademica/anais/6mostra/5/371.pdf $>$ Acesso em: 05 maio 2016.

FALKENMARK, Malin. The massive water scarcity now threatening Africa: why isn't it being addressed? Ambio, v. 18, n. 2, p. 112-118, 1989.

FOOD AND AGRICULTURE ORGANIZATION OF THE UNITED NATIONS. Aquastat. Available at: <http://www.fao.org/nr/water/aquastat/main/index. stm>. Accessed: 05 May 2016.

GOMES, Natália Fernanda. A regulação internacional dos subsídios agrícolas: a contemporaneidade do paradigma realista para a compreensão do sistema de comércio agrícola internacional vigente. Revista de Direito Internacional, v.10, n. 1, jun. 2013. doi:10.5102/rdi. v10i1.2067.

GULBEKIAN, Think Thank. Water and the future of humanity: revisiting water security. New York: Springer, 2013.

HOEKSTRA, Arjen Y. The relation between international trade and freshwater scarcity, Jan. 2010. Available at: <https:/ / www.wto.org/english/res_e/reser_e/ersd201005_e. pdf $>$ Accessed: 17 Apr. 2016.

HOEKSTRA, Arjen Y.; CHAPAGAIN, Ashok K. Globalization of water: sharing the planet's freshwater resources. Malden: Wiley-Blackwell, 2008.

HOEKSTRA, Arjen Y.; CHAPAGAIN, Ashok K.; ALDAYA, M.M.; MEKONNEN, M. M. Water footprint assessment manual: the global standard. Avaiable at: </en/ resources/publications/water-footprint-assessmentmanual-global-standard/>. Accessed: 6 May 2016.

HOEKSTRA, Arjen Y.; HUNG, P. Q. Virtual water trade: a quantification of virtual water flows between nations in relation to international crop trade. Value of Water Research Report Series, n. 11, p. 25-47, Sept. 2002.

HOMER-DIXON, Thomas. The ingenuity gap: facing the economic, environmental, and other challenges of an increasingly complex and unpredictable future. New York: Vintage, 2002.

HORLEMANN, Lena; NEUBERT, Susanne. Virtual water trade: a realistic concept for resolving the water crisis? Bonn: Deutsches Institut für Entwicklungspolitik, 2007.

LOBO, Thaís. Brasil exporta cerca de 112 trilhões de litros de água doce por ano. O Globo, 11 set. 2012. Ciência. Disponível em: <http://oglobo.globo.com/sociedade/ciencia/brasil-exporta-cerca-de-112-trilhoes-delitros-de-agua-doce-por-ano-6045674>. Acesso em: 17 abr. 2016.

NASH, John; MCCALLA, Alex. Reforming agricultural trade for developing countries: key issues for a pro-development 
outcome of the Doha round. Washington: World Bank Publications, 2006.

O'HEAR, Anthony. Karl Popper: philosophy and problems. New York: Cambridge University Press, 1996.

RAYNOLDS, Laura T.; MURRAY, Douglas; HELLER, Andrew. Regulating sustainability in the coffee sector: a comparative analysis of third-party environmental and social certification initiatives. Agriculture and human values, v. 24, n. 2, p. 147-163, Mar. 2007. doi:10.1007/s10460006-9047-8.

REBOUÇAS, Aldo da Cunha; Tundisi, José Galizia (Coord.). Águas doces no Brasil. São Paulo: Escrituras, 1999.

SÃO PAULO. Secretaria do Meio Ambiente. Projeto ambiental estratégico aquiferos: síntese das atividades período 2007-2010. São Paulo: Instituto Geológico, 2011. (Cadernos do Projeto Ambiental Estratégico Aquíferos, n. 3) Disponível em: <http://www.igeologico.sp.gov.br/ downloads/livros/Sintese\%20Aquiferos.pdf $>$ Acesso em: 05 maio 2016.

SOARES, Guido Fernando Silva. Direito Internacional do Meio Ambiente: emergência, obrigações e responsabilidades. São Paulo: Atlas, 2003.

TECLAFF, Ludwik A. The river basin in history and law. Haia: Martinus Nijhoff, 1967.

TELLES, D. D’A. Água na agricultura e pecuária. In: REBOUÇAS, A; Braga B; TUNDISI, J. G. (Orgs). Águas doces no Brasil: capital ecológico, uso e conservação. 2. ed. São Paulo: Escrituras, 1999.
UNITED NATIONS WORLD WATER ASSESSMENT PROGRAMME. Water for people, water for life. New York: Berghahn Books, 2003.

UNITED NATIONS. DEPARTMENT OF ECONOMIC AND SOCIAL AFFAIRS. STATISTICS DIVISION. UN Comtrade Database, 2016. Available: <http:/ / comtrade.un.org/data/> Accessed: 05 May 2016.

WARNER, Jeroen; SEBASTIAN, Antoinette; EMPINOT'TI, Vanessa. Claiming (back) the land: the geopolitics of egyptian and south african land and water grabs. Ambiente \& Sociedade. v. 16, n. 2, p. 1-24, June 2013. doi:10.1590/S1414-753X2013000200002.

WEISS, Edith Brown; JACKSON, John H.; BERNASCONI-OSTERWLADER, Nathalie. Reconciling environment and trade. Leiden: Brill, 2008.

WEISS, Edith Brown; SLOBODIAN, Lydia. Virtual Water, Water Scarcity, and International Trade Law. Journal of International Economic Law, v. 17, n. 4, p. 717737, Dec. 2014. doi:10.1093/jiel/jgu038.

WORLD TRADE ORGANIZATION. United States: measures concerning the importation, marketing and sale of tuna and tuna products, 2012. Available at: <https://www.wto.org/english/tratop_e/ dispu_e/381abr_e.pdf $>$. Accessed: 05 May 2016.

WORLD TRADE ORGANIZATION. World Trade Report 2010: Trade in natural resources, 2010. Available at: <https://www.wto.org/english/res_e/publications_e/ wtr10_e.htm> Accessed: 05 May 2016. 
Anexo A: a água virtual em alimentos

COMUNS
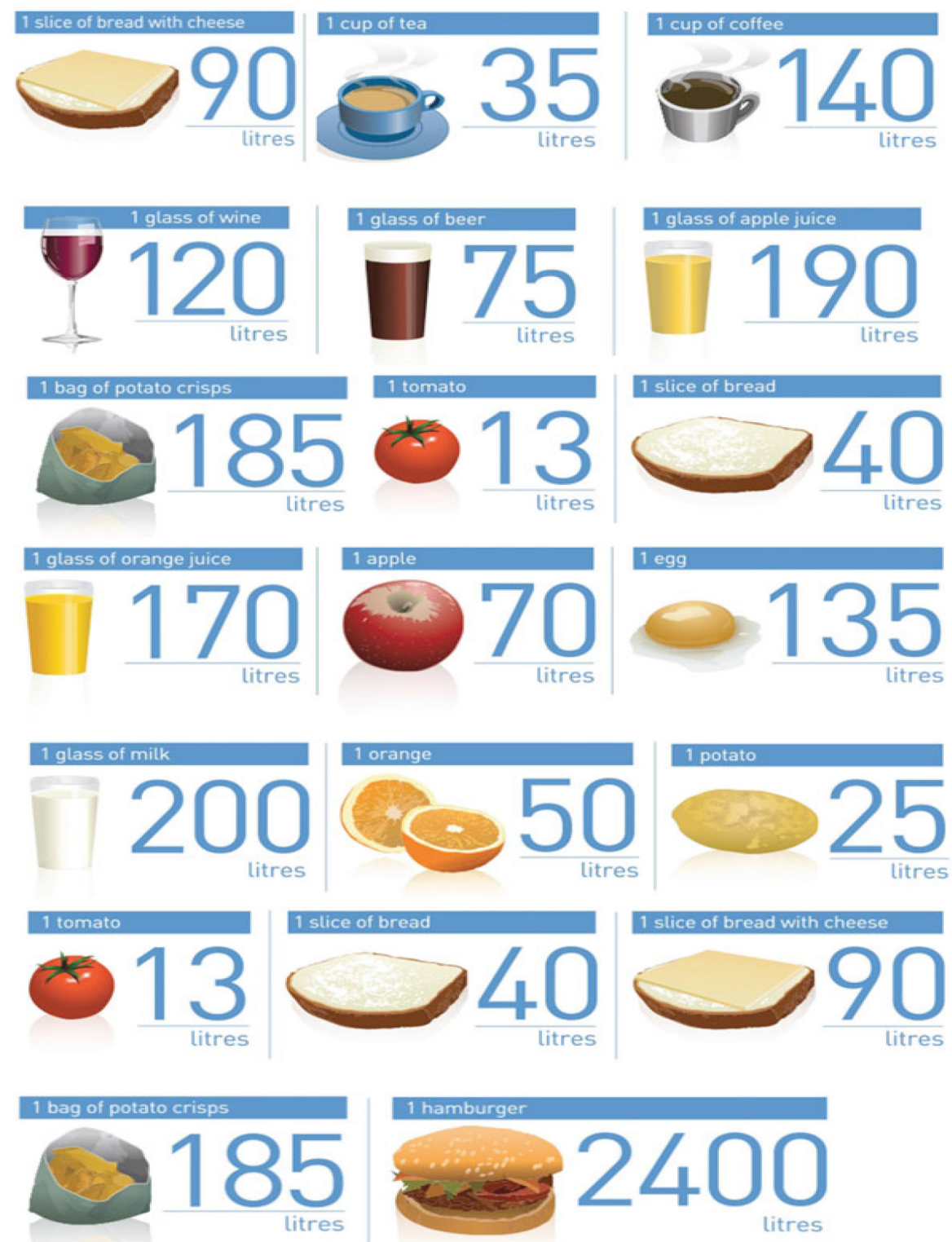

Fonte: FAO, 2012; editado pela FAO WATER 
Para publicar na Revista de Direito Internacional, acesse o endereço eletrônico www.rdi.uniceub.br ou www.brazilianjournal.org.

Observe as normas de publicação, para facilitar e agilizar o trabalho de edição. 